\title{
On Acetyl-CoA as a Gauge of Cellular Metabolic State
}

\author{
L. CAI AND B.P. TU \\ Department of Biochemistry, UT Southwestern Medical Center, Dallas, Texas 75390-9038 \\ Correspondence: benjamin.tu@utsouthwestern.edu
}

\begin{abstract}
Many activities within a cell must be intimately coordinated with its metabolic state. Understanding these connections to metabolism is critical to deciphering the regulation of a variety of cellular processes. However, despite intensive research in these areas, the precise mechanisms by which a cell monitors its metabolic state remain controversial and poorly understood. Herein, we discuss the thesis that intracellular levels of the metabolite acetyl-CoA represent a critical gauge of cellular metabolic state, which is cleverly monitored by the cell through dynamic acetylation modifications to direct a variety of outputs connected to energy metabolism, cell growth, and survival.
\end{abstract}

Acetyl-CoA is a central metabolite that is involved in many metabolic transformations within the cell. It is a molecule of acetate joined to coenzyme A via a thioester linkage. In essence, it is an activated two-carbon unit of acetate that the cell uses to stitch together numerous metabolites such as fatty acids and sterols. Alternatively, the acetyl group of acetyl-CoA can be oxidized by way of the TCA cycle to produce reduced NADH and $\mathrm{FADH}_{2}$, which subsequently fuel ATP production through the electron transport chain. Acetyl-CoA also functions as the acetyl group donor for metabolite and protein acetylation modifications. Although previously thought to occur primarily on lysine residues present on histone tails, protein acetylation has recently emerged as a key posttranslational modification that can also occur on many nonhistone proteins to regulate numerous cellular and metabolic processes (Polevoda and Sherman 2002; Kim et al. 2006; Choudhary et al. 2009; Wang et al. 2010; Zhao et al. 2010). Given the immediate link between acetyl-CoA and central carbon metabolism, it is predictable that protein acetylation modifications might represent a means to posttranslationally regulate particular protein activities in tune with metabolism, possibly through intracellular acetyl-CoA fluctuations (Albaugh et al. 2011). However, despite many investigations into the role of acetylation modifications in various cellular pathways, few have convincingly shown how and why particular proteins might be acetylated in response to cellular metabolic state.

Using the yeast metabolic cycle (YMC) system wherein a highly synchronized yeast cell population continuously transitions between growth and quiescent-like phases (Laxman and $\mathrm{Tu}$ 2010; Tu 2010), we recently uncovered a direct role for acetyl-CoA in the regulation of specific protein acetylation modifications in budding yeast (Cai et al. 2011). These and other recent findings, discussed below, support the view that a primordial role of protein acetylation may have been to enable the coordination of numerous cellular activities with the metabolic status of the cell (Takahashi et al. 2006; Choudhary et al. 2009; Lin et al. 2009; Wellen et al. 2009; Wang et al. 2010; Zhao et al. 2010; Cai et al. 2011). They further predict that intracellular acetyl-CoA fluctuations might represent a distinctive gauge of cellular metabolic state that could be decoded by way of dynamic acetylation and deacetylation reactions.

\section{PROTEIN ACETYLATION AND ITS IMPORTANCE}

\section{Acetylation of Proteins}

Not only does acetyl-CoA lie at the intersection of various metabolic pathways, it also provides the acetyl group for numerous protein acetylation reactions (Saxholm et al. 1982). Thus, acetyl-CoA is ideally situated to link metabolic status to the control of cellular activities. The acetylation of proteins has been found to occur primarily on the amino terminus of proteins as well as on the $\varepsilon$-amino group of lysine residues within proteins.

$N$ - $\alpha$-acetylation, an irreversible modification that occurs during translation, is pervasive in eukaryotes. It has been estimated to occur in $\sim 50 \%$ of yeast proteins and $80 \%-90 \%$ of mammalian cell proteins (Polevoda and Sherman 2003). However, the cellular role of this ubiquitous modification remains poorly understood (Arnesen 2009). Given its dependence on acetyl-CoA, it is plausible to speculate that this common modification of newly translated proteins could be tied to cellular metabolic state in some manner.

Protein acetylation can also take place on the $\varepsilon$-amino group of lysine residues. Since the discovery of histone acetylation in the 1960s (Allfrey et al. 1964), myriad proteins have been found to be modified by lysine acetylation with the improvement of mass spectrometry technologies (Polevoda and Sherman 2002; Kim et al. 2006; 
Choudhary et al. 2009; Zhang et al. 2009; Wang et al. 2010; Zhao et al. 2010). Collectively, these studies have shown that thousands of proteins in a variety of cellular pathways can be modified by lysine acetylation. In some cases, virtually every enzyme of a particular metabolic pathway can be acetylated (Zhao et al. 2010). The physiological role and underlying logic of such modifications are currently being investigated.

\section{Regulation of Protein Acetylation by Deacetylases}

The importance of protein acetylation modifications in the cell has been frequently revealed from studies of protein deacetylases. The current emphasis on the regulation of protein acetylation has been on the deacetylation side, with intense interest in the nicotinamide adenine dinucleotide $\left(\mathrm{NAD}^{+}\right)$-dependent class of deacetylases called sirtuins (Blander and Guarente 2004). Interestingly, the catalytic mechanism of deacetylation by sirtuins is distinct from other deacetylases (Imai et al. 2000a,b). The sirtuins couple deacetylation with hydrolysis of $\mathrm{NAD}^{+}$, which yields $O$-acetyl-ADP-ribose and nicotinamide as products of the catalytic reaction (Tanner et al. 2000; Tanny and Moazed 2001). The reaction is therefore dependent on $\mathrm{NAD}^{+}$as a substrate and thus been proposed to be regulated by the intracellular $\mathrm{NAD}^{+} /$ NADH ratio or $\mathrm{NAD}^{+}$availability (Fulco et al. 2003; Blander and Guarente 2004). Moreover, the sirtuins are also subject to inhibition by nicotinamide (Bitterman et al. 2002).

Sirtuins are well known for their roles in mediating extension of life span under conditions of caloric restriction (Blander and Guarente 2004). The enzymes are conserved from bacteria to humans (Brachmann et al. 1995). Their impact on longevity is also consistently observed for different model organisms (Tissenbaum and Guarente 2001; Rogina and Helfand 2004). In budding yeast, where sirtuins were first discovered (Rine and Herskowitz 1987), activated Sir2 during growth in limited carbon sources reduces extrachromosomal rDNA circles by silencing the rDNA chromatin (Sinclair and Guarente 1997; Kaeberlein et al. 1999). In worms, SIR-2.1 interacts with 14-3-3 protein to activate the forkhead transcription factor DAF-16 that drives expression of stress response genes to combat aging (Berdichevsky et al. 2006).

In mammals, there exist seven sirtuins and they appear to be prominent regulators of a variety of metabolic processes. The closest Sir2 homolog, Sirt1, localizes to the nucleus and cytoplasm (Michan and Sinclair 2007; Tanno et al. 2007). It can down-regulate adipogenesis and up-regulate fat mobilization by repressing transcription of PPAR- $\gamma$ in white adipose tissue (Picard et al. 2004), and activate gluconeogenesis in the liver by deacetylating PGC1- $\alpha$ (Rodgers et al. 2005). Sirt1 can also inhibit cell death and increase stress resistance by deacetylating or interacting with transcription factors, including $\mathrm{p} 53$, FOXO, and E2F (Luo et al. 2001; Vaziri et al. 2001; Brunet et al. 2004; Wang et al. 2006; Chen and Guarente 2007). Recent evidence from mouse models indicates that Sirt1 exerts a protective effect on obesity, diabetes, and fatty liver disease (Herranz and Serrano 2010). The mitochondrial sirtuin Sirt3 also impacts a number of metabolic processes, including ketone body synthesis, the urea cycle, and fatty acid oxidation (Hallows et al. 2006, 2011; Hirschey et al. 2010). Collectively, studies of sirtuin knockout phenotypes have revealed that compromising the function of this class of deacetylation enzymes can result in a variety of metabolic consequences and disorders, highlighting the importance of proper protein acetylation regulation.

\section{ACETYL-COA LINKS PROTEIN ACETYLATION TO METABOLIC STATE}

\section{Protein Acetylation Regulated by Acetyl-CoA Levels}

In light of the numerous studies that have focused on the role of protein deacetylases, it should be noted that these deacetylases ultimately act on acetylated protein substrates. How do these proteins become acetylated in the first place? The level of acetylation on a particular protein is the net result of acetylation and deacetylation reactions. These reactions need to be dynamic enough to rapidly respond to the appropriate regulatory signals and cues. For example, it has been demonstrated that the turnover of histone acetylation can occur within minutes (Moore et al. 1979). Nevertheless, these two opposing reactions should in theory work in a complementary manner to avoid futile cycles. How then does the cell know when to acetylate a particular substrate? How is the regulation of protein acetylation executed from the acetylation side of the reaction?

Classical studies of transcription have shown that transcription factors can recruit acetyltransferase-containing coactivator complexes to DNA to catalyze histone acetylation, which consequently facilitates gene activation (Lee and Workman 2007). Thus, recruitment of acetyltransferase enzymes to the substrate to be acetylated is one typical mechanism through which acetylation reactions could be regulated (Fig. 1). Alternatively, the
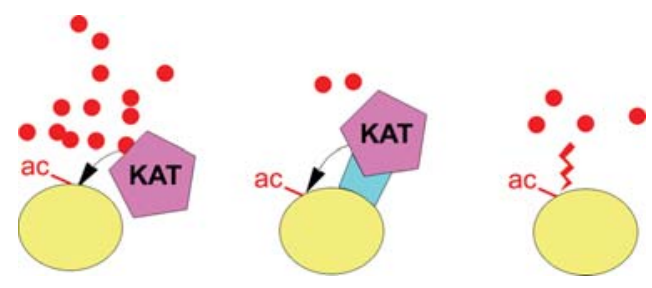

Figure 1. Possible regulatory mechanisms for protein acetylation. Certain lysine acetyltransferases (KATs) may be regulated by the availability of acetyl-coenzyme A (CoA); such reactions are tightly coupled to the metabolic status of the cell. Other protein acetylation events may be controlled by recruitment of KATs to their substrates through adaptor proteins. Protein acetylation can also occur spontaneously without enzyme catalysis. Acetyl-CoA molecules are depicted as red dots. ac, acetylated lysine. 
acetylation reaction could be regulated by the availability of acetyl-CoA, which functions as the acetyl donor (Fig. 1). Such a link between protein acetylation and intracellular acetyl-CoA pools was first demonstrated in budding yeast. Yeast cells lacking the two acetyl-CoA synthetase enzymes ACS1 and ACS2 exhibit severely reduced levels of bulk histone acetylation (Takahashi et al. 2006). Moreover, a temperature sensitive mutant in ACS2 exhibited synthetic growth phenotypes in combination with mutations in acetyltransferase enzymes (Takahashi et al. 2006). Thus, it became apparent that the metabolic enzymes that control acetyl-CoA biosynthesis also supply the nuclear acetyl-CoA pools required for histone acetylation. Bulk histone acetylation was also shown to be responsive to glucose availability in yeast (Friis et al. 2009).

\section{Acetyl-CoA Links Cell Growth and Proliferation to Metabolic State in Yeast}

We recently showed that intracellular acetyl-CoA levels could actually function as a direct regulator of certain protein acetylation reactions (Cai et al. 2011; Fig. 2). Yeast cells can become highly synchronized during continuous growth under nutrient-limited conditions (Laxman and Tu 2010; Tu 2010). The cell population undergoes robust oscillations in oxygen consumption and alternates between growth and quiescent phases in these "yeast metabolic cycles" (YMC) (Tu et al. 2005). There are three phases in the YMC that we term OX (oxidative), RB (reductive, building), and RC (reductive, charging). The OX phase is analogous to a growth phase. When oxygen consumption increases during this temporal window, cells activate the transcription of growth genes, including those involved in ribosome biogenesis, amino acid biosynthesis, translation, and the maturation of rRNA and tRNAs. Virtually every gene that is induced during this time can be predicted to have some function that is important for growth (Tu et al. 2005; Tu and McKnight 2006; Cai et al. 2011). In the RB phase, cell division occurs as cells significantly decrease their oxygen consumption and express genes involved in cell cycle control and progression. In the $\mathrm{RC}$ phase, when oxygen consumption remains low, cells express many genes associated with stress, survival, and slow growth (Brauer et al. 2008), including heat shock response, vacuole, autophagy, ubiquitin-proteasome, and various detoxification genes. They also accumulate the storage carbohydrates trehalose and glycogen in a manner reminiscent of stationary phase cells (Shi et al. 2010). Hence, this temporal window can be likened to stationary phase or quiescence.

We observed that addition of carbon sources such as glucose or fermentation products like ethanol and acetate could induce cells from the quiescent-like RC phase of the YMC to enter growth (Cai et al. 2011). This is accompanied by a sharp increase in intracellular acetyl-CoA levels. Although exogenous carbon sources are converted to acetyl-CoA, they also stimulate production of acetylCoA from endogenous carbon sources to further amplify the response. The induction of acetyl-CoA levels during growth reflects its active engagement in metabolic reactions. Moreover, we also observed that acetyl-CoA levels in the OX growth phase of an uninterrupted YMC are about sixfold higher than in the RC quiescent phase $(\mathrm{Tu}$ et al. 2007; Cai et al. 2011). Likewise, in batch culture,

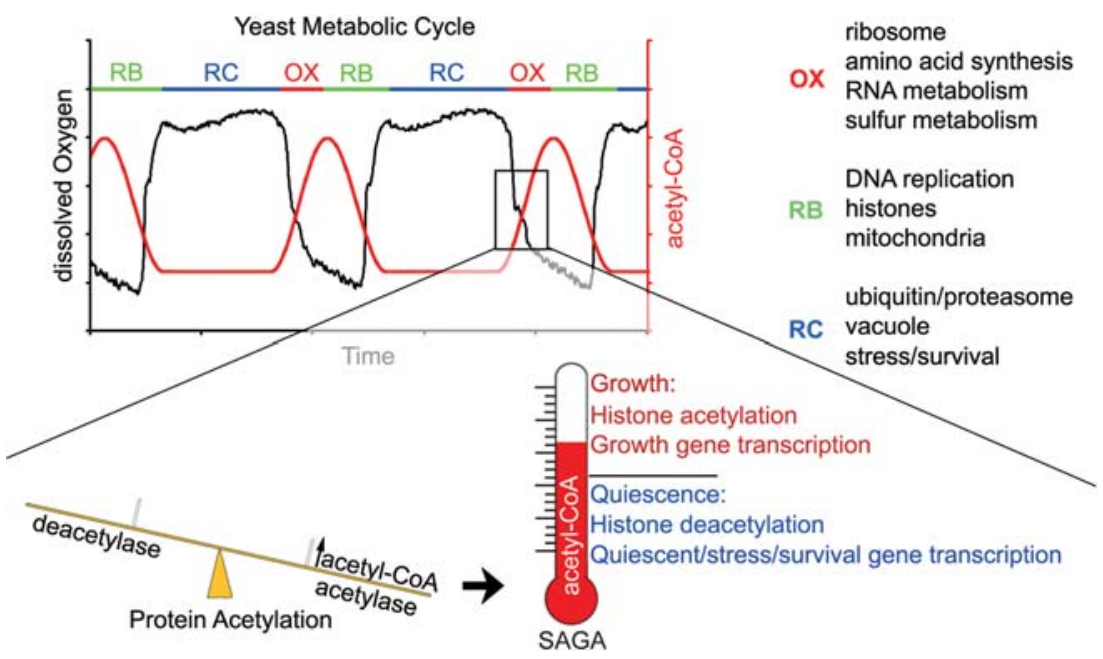

Figure 2. Acetyl-CoA drives the transcription of genes important for growth. Yeast cells undergo robust oscillations in oxygen consumption during continuous growth. They transition between OX (oxidative), RB (reductive, building), and RC (reductive, charging) phases during each metabolic cycle and each phase is characterized by distinct gene expression profiles. The OX phase transcripts predominantly comprise genes involved in growth. During this time, cells increase oxygen consumption and experience a surge in acetyl-CoA levels. The acetylation status of a protein is regulated by both deacetylases and acetylases. Rising acetyl-CoA levels can tip the balance toward acetylation through an acetyltransferase that is more responsive to acetyl-CoA levels, such as Gcn5p. Gcn5p/SAGA was recently shown to catalyze histone acetylation primarily on growth genes to activate their transcription (Cai et al. 2011). 
acetyl-CoA levels decrease as the culture transitions from $\log$ to stationary phase.

To test the possibility that some protein acetylation events might be responsive to acetyl-CoA levels, we conducted a proteomic screen to identify acetylated peptides in cells entering growth induced by addition of ${ }^{13}$ C-labeled acetate (Cai et al. 2011). From a list of candidates, we confirmed that three components Spt7p, Sgf73p, and Ada3p, of the multisubunit transcriptional activator SAGA are dynamically acetylated in tune with acetyl-CoA levels. They are directly acetylated by Gcn5p, the catalytic acetyltransferase present within SAGA (Grant et al. 1997). From the screen, we also noticed a large number of histone peptides containing a ${ }^{13} \mathrm{C}$-labeled acetyl group. Some of these sites are known to be acetylated by SAGA (Grant et al. 1999). We examined acetylation of K9, K14, K18, K23, K27 on $\mathrm{H} 3$ and $\mathrm{K} 5, \mathrm{~K} 8, \mathrm{~K} 12$ on $\mathrm{H} 4$ and confirmed that they are dynamically acetylated only when cells enter the growth phase of the YMC, in tune with the increase in acetylCoA. In contrast, acetylation of $\mathrm{H} 3 \mathrm{~K} 56$, which is a mark associated with cell division (Masumoto et al. 2005; Xu et al. 2005), increased at later time points during $\mathrm{RB}$ phase precisely when cells initiated the division process.

To study whether dynamic histone acetylation drives transcriptional regulation in response to carbon source availability, we performed chromatin immunoprecipitation analysis of many of these marks at selected genes known to be regulated by SAGA. We found that histone acetylation robustly increased on the growth genes at OX phase but not for genes in RC phase, suggesting that the surge of acetyl-CoA production during OX phase specifically drives growth gene transcription, whereas stress and survival genes are less dependent on histone acetylation for their activation in RC phase (Cai et al. 2011). We then surveyed the genome-wide occupancy of H3K9 acetylation and SAGA during the growth (OX) and quiescent-like (RC) phases of the YMC. Strikingly, significant regions of $\mathrm{H} 3 \mathrm{~K} 9$ acetylation were present during growth, almost exclusively at the growth genes (e.g., ribosome biogenesis, translation, amino acid synthesis). Many of these sites of H3K9ac correlate well with those occupied by SAGA. In contrast, H3K9ac peaks were very sparse during the quiescent-like RC phase despite significant binding of SAGA to the quiescent phase genes. Taken together, these data reveal that the transcription of genes important for cell growth is driven by histone acetylation that is induced upon a burst of acetyl-CoA production.

Our study also suggests that only certain acetyltransferases might be regulated by acetyl-CoA fluctuations (Cai et al. 2011). All the dynamically acetylated nonhistone proteins identified in our study thus far appear to be Gcn5 substrates. The dynamic acetylation of H4 catalyzed by the NuA4 complex is also dependent on Gen5. Moreover, gcn5 $\Delta$ mutants exhibit slow growth and cannot undergo the bursts of respiratory growth that are a hallmark of the YMC (Cai et al. 2011). Therefore, we hypothesize that Gen5 is the key acetyltransferase that regulates protein acetylation in response to acetyl-CoA levels in yeast (Fig. 2).

\section{ACETYL-COA-DRIVEN REGULATION OF ACETYLATION IN MAMMALIAN CELLS?}

Could acetyl-CoA also be driving the acetylation of particular proteins in mammalian cells, where nutrient uptake and growth control mechanisms could be regulated quite differently compared with single-celled organisms (Vander Heiden et al. 2009)? There are now several examples that suggest that acetyl-CoA is just as likely to play a critical role in regulating key protein acetylation reactions in mammalian cells.

\section{ATP Citrate Lyase and the Regulation of Histone Acetylation}

In mammalian cells, nucleocytosolic pools of acetylCoA are thought to be primarily supplied by glucosederived citrate through ATP citrate lyase (ACLY) (Wellen et al. 2009). The TCA cycle intermediate citrate can move from the mitochondria to the cytosol where it can be converted to oxaloacetate and acetyl-CoA by ACLY (Srere 1959). Knockdown of ACLY by RNAi leads to a decrease in global histone acetylation and inhibits the expression of several metabolic genes, supporting the idea that acetyl-CoA levels could regulate gene transcription through modulating histone acetylation in mammalian cells (Wellen et al. 2009).

\section{Regulation of PGC-1 $\alpha$ by Gen5 and Sirt1}

A second example in mammalian cells where acetylCoA may regulate a key acetylation reaction comes from studies of the peroxisomal proliferator-activated receptor $\gamma$ coactivator $1-\alpha(\mathrm{PGC}-1 \alpha)$. PGC- $1 \alpha$ is a key regulator of mitochondrial activity in mammals $(\mathrm{Wu}$ et al. 1999; Jeninga et al. 2010). It interacts with different transcription factors in different organs to regulate transcription of metabolic genes required for energy homeostasis (Jeninga et al. 2010). PGC- $1 \alpha$ modulates expression of genes involved in fatty acid oxidation and fatty acid synthesis, glycolysis, and gluconeogenesis (Puigserver et al. 1998; Yoon et al. 2001). Reversible acetylation of PGC- $1 \alpha$ has emerged as an important regulatory mechanism that signals energy demand that in turn controls the transcription of metabolic enzymes. Under starvation conditions, PGC- $1 \alpha$ is turned on by Sirt1-catalyzed deacetylation (Gerhart-Hines et al. 2007). Under energy-sufficient conditions, PGC- $1 \alpha$ is acetylated by GCN5, which leads to its inactivation (Lerin et al. 2006). In the case of the metabolic regulation of this coactivator, it is conceivable that, when sufficient nutrients are supplied, elevated acetyl-CoA levels could drive the acetylation of PGC- $1 \alpha$ to repress its activity and turn off the starvation response. Thus, the activity of this coactivator appears to be under the control of 
reciprocal regulation by dynamic acetylation and deacetylation (Dominy et al. 2010).

\section{WHAT IS THE LOGIC BEHIND THE THOUSANDS OF NEWLY IDENTIFIED ACETYLATED PROTEINS?}

In recent years, protein acetylation has been linked to almost every cellular or regulatory pathway (Kim et al. 2006; Choudhary et al. 2009; Wang et al. 2010; Zhao et al. 2010). Genetic knockouts of HDACs and sirtuins have been reported to exhibit a wide variety of interesting and elaborate metabolic phenotypes. As the number of acetylated proteins appears to keep increasing, is there a unifying logic that might explain how each of these modifications could be coordinated with metabolism and metabolic state? Moreover, protein lysine residues can also be succinylated or propionylated (Cheng et al. 2009; Zhang et al. 2011). For many of these identified posttranslational modifications, it is unclear what percentage of the protein is actually modified. With the improved sensitivity of mass spectrometry technologies, a small percentage of the total amount of an abundant peptide could be acetylated and it might still be identified as a positive hit.

Based on chemical principles it is well documented that primary amines are nucleophilic and can react with thioesters spontaneously in the absence of enzymatic catalysis (Deletre and Levesque 1990; Castro 2009). Thus, the $\varepsilon$-amino group of the lysyl side chain could react spontaneously with acetyl-CoA at slow rates to produce acetyllysine (Fig. 1). In support of this idea, spontaneous acetylation of histones in the absence of acetyltransferase enzyme has been documented in assays monitoring histone acetylation (Tanner et al. 1999; Berndsen and Denu 2005). Likewise, lysines could also react nonenzymatically with other CoA derivatives, such as succinylCoA or propionyl-CoA. Based on these considerations, caution should be taken when investigating some of these lysine posttranslational modifications as, while some of them could indeed be regulatory, others might actually be nonspecific. Moreover, particular lysine residues within a protein might become more nucleophilic because of local hydrogen bonding or salt-bridge interactions, thus making them more susceptible to nonspecific acylation by CoA metabolites. Thus, rigorous experimentation will be needed to establish which acetylation modifications are physiologically relevant for regulation of protein function.

\section{ACETYLATION OF HISTONES TO ENABLE COORDINATION OF GROWTH GENE EXPRESSION WITH METABOLIC STATE}

Our studies using the yeast metabolic cycle have revealed that specific proteins are acetylated in tune with acetyl-CoA levels, whereas others can be acetylated independent of acetyl-CoA fluctuations (Cai et al. 2011). In particular, multiple lysines on histone $\mathrm{H} 3$ are acetylated by Gen5 in response to a burst of acetyl-CoA production to direct the activation of growth genes as cells exit quiescence and transition into growth (Fig. 2). These data suggest that histone acetylation was designed to enable a cell to coordinate the expression of a distinct class of genes with carbon source availability. Because of its central role in biosynthesis and energy production, a cell may have chosen to monitor its ability to produce acetyl-CoA to assess whether it can complete a round of growth and division. Thus, these considerations suggest that a primordial role of protein acetylation was to enable a cell to regulate cellular activities in tune with metabolic state. Eventually, some acetylation modifications might have been decoupled from acetyl-CoA fluctuations and instead could be driven by recruitment or access to the acetyltransferase enzyme (Fig. 1).

One reason that this mode of acetylation regulation by acetyl-CoA may have been overlooked is because of our tendency to culture cells in nutrient-rich media that enable cells to persist in a rapid proliferative state where one cell division happens right after another. Acetyl-CoA levels are consistently high when glucose concentrations are abundant, therefore histones and other proteins may be constitutively acetylated and this mode of regulation could be masked. However, in reality for the life of a microbial cell or for cells within a cancerous tumor, carbon source availability could be limiting or unpredictable, and thus acetyl-CoA fluctuations could play a critical role in driving particular acetylation reactions that regulate growth and proliferation.

\section{A GENERAL DETOXIFICATION ROLE FOR PROTEIN DEACETYLASES TO ELIMINATE ABERRANT ACETYLATION?}

It seems quite sensible for cells to use acetyl-CoA and protein acetylation to regulate protein functions in tune with metabolic state. However, the selection pressure for increased intracellular acetyl-CoA (and other acylCoA metabolites) to promote rapid cell proliferation may have also led to a concomitant increase in the rate of nonspecific acetylation of proteins (Fig. 1). Such spontaneous acetylation of proteins could be undesirable and contribute to protein misfolding, dysfunction, or aggregation and compromise the ability of a cell to function normally. Consequently, the cell would be in need of a surveillance system to remove these nonspecific modifications from proteins. Thus, it is predictable that some of the deacetylases might play a key role in removing aberrant acetylation modifications in proteins as part of a "clean-up" or "detoxification" function. In support of this idea, although a number of mitochondrial proteins are acetylated and there are sirtuins present within the mitochondria, thus far a mitochondria-localized acetyltranferase has not yet been identified (Huang et al. 2010). Moreover, it is unclear whether there are enzymes that can specifically catalyze lysine succinylation or propionylation (Cheng et al. 2009; Zhang et al. 2011). Therefore, the absence of particular deacetylases may then result in an increased frequency of aberrant acetylation 
modifications on proteins, which could contribute toward a variety of detrimental metabolic phenotypes and perhaps shortened life span. Future studies will be required to resolve which of these modifications are truly regulatory as opposed to coincidental.

\section{CONCLUSIONS}

The activated two-carbon unit of acetyl-CoA is a fundamental building block and energy source for cell growth and proliferation. Acetyl-CoA levels increase during growth, which consequently induces histone acetylation to enable the transcription of growth genes. As a result, acetyl-CoA could be the key regulator of cell growth and proliferation through the modulation of particular protein acetylation reactions, especially those at histones in chromatin. It is now predictable that a primordial function of protein acetylation may have been to enable a cell to precisely regulate a variety of protein functions in tune with carbon source availability and thus its metabolic state. High acetyl-CoA levels would signify a "proliferative" or "fed" state, while low acetylCoA levels would be indicative of a "quiescent" or "starved" state. However, use of such an acetylationbased mechanism for protein regulation may have come with a consequence in that particular lysine residues might be more prone to nonenzymatic acetylation, eliciting the need for a cleanup or detoxification system that could be provided by deacetylases. With the realization that glucose availability is linked to acetyl-CoA production, and given the wealth of metabolic phenotypes that have been linked to sirtuins and HDACs, collectively these considerations suggest that a dynamic interplay between acetylation and deacetylation could represent the basis for the regulation of numerous cellular and metabolic processes. Thus, there is now compelling evidence to support the view that acetyl-CoA represents a fundamental and widely conserved gauge of cellular metabolic state.

\section{ACKNOWLEDGMENTS}

The authors acknowledge the funding support from the University of Texas Southwestern Endowed Scholars Program, Burroughs Wellcome Fund, award R01GM094314 from the National Institute of General Medical Sciences, the David and Lucile Packard Foundation, the Damon Runyon Cancer Research Foundation (B.P.T.), and a Frank and Sara McKnight Graduate Fellowship (L.C.).

\section{REFERENCES}

Albaugh BN, Arnold KM, Denu JM. 2011. KAT(ching) metabolism by the tail: Insight into the links between lysine acetyltransferases and metabolism. ChemBioChem 12: 290-298.

Allfrey VG, Faulkner R, Mirsky AE. 1964. Acetylation and methylation of histones and their possible role in the regulation of RNA synthesis. Proc Natl Acad Sci 51: 786-794.

Arnesen T. 2009. Protein N-terminal acetylation: NAT 20072008 Symposia. BMC Proc 3: S1.
Berdichevsky A, Viswanathan M, Horvitz HR, Guarente L. 2006. C. elegans SIR-2.1 interacts with 14-3-3 proteins to activate DAF-16 and extend life span. Cell 125: $1165-$ 1177.

Berndsen CE, Denu JM. 2005. Assays for mechanistic investigations of protein/histone acetyltransferases. Methods 36: $321-331$.

Bitterman KJ, Anderson RM, Cohen HY, Latorre-Esteves M, Sinclair DA. 2002. Inhibition of silencing and accelerated aging by nicotinamide, a putative negative regulator of yeast sir2 and human SIRT1. J Biol Chem 277: 45099-45107.

Blander G, Guarente L. 2004. The Sir2 family of protein deacetylases. Annu Rev Biochem 73: 417-435.

Brachmann CB, Sherman JM, Devine SE, Cameron EE, Pillus L, Boeke JD. 1995. The SIR2 gene family, conserved from bacteria to humans, functions in silencing, cell cycle progression, and chromosome stability. Genes Dev 9: 28882902.

Brauer MJ, Huttenhower C, Airoldi EM, Rosenstein R, Matese JC, Gresham D, Boer VM, Troyanskaya OG, Botstein D. 2008. Coordination of growth rate, cell cycle, stress response, and metabolic activity in yeast. Mol Biol Cell 19: 352-367.

Brunet A, Sweeney LB, Sturgill JF, Chua KF, Greer PL, Lin Y, Tran H, Ross SE, Mostoslavsky R, Cohen HY, et al. 2004. Stress-dependent regulation of FOXO transcription factors by the SIRT1 deacetylase. Science 303: 2011-2015.

Cai L, Sutter BM, Li B, Tu BP. 2011. Acetyl-CoA induces cell growth and proliferation by promoting the acetylation of histones at growth genes. Mol Cell 42: 426-437.

Castro EA. 2009. Kinetics and mechanism of the aminolysis of thioesters and thiocarbonates in solution. Pure Appl Chem 81: 685-696.

Chen D, Guarente L. 2007. SIR2: A potential target for calorie restriction mimetics. Trends Mol Med 13: 64-71.

Cheng Z, Tang Y, Chen Y, Kim S, Liu H, Li SS, Gu W, Zhao Y. 2009. Molecular characterization of propionyllysines in nonhistone proteins. Mol Cell Proteomics 8: 45-52.

Choudhary C, Kumar C, Gnad F, Nielsen ML, Rehman M, Walther TC, Olsen JV, Mann M. 2009. Lysine acetylation targets protein complexes and co-regulates major cellular functions. Science 325: $834-840$.

Deletre M, Levesque G. 1990. Kinetics and mechanism of polythioamidation in solution 0.1 . Reaction of mono(dithioester)S and bis(dithioester)S with excess amine. Macromolecules 23: 4733-4741.

Dominy JE Jr, Lee Y, Gerhart-Hines Z, Puigserver P. 2010. Nutrient-dependent regulation of PGC-1 $\alpha$ 's acetylation state and metabolic function through the enzymatic activities of Sirt1/GCN5. Biochim Biophys Acta 1804: 1676-1683.

Friis RM, Wu BP, Reinke SN, Hockman DJ, Sykes BD, Schultz MC. 2009. A glycolytic burst drives glucose induction of global histone acetylation by picNuA4 and SAGA. Nucleic Acids Res 37: 3969-3980.

Fulco M, Schiltz RL, Iezzi S, King MT, Zhao P, Kashiwaya Y, Hoffman E, Veech RL, Sartorelli V. 2003. Sir2 regulates skeletal muscle differentiation as a potential sensor of the redox state. Mol Cell 12: 51-62.

Gerhart-Hines Z, Rodgers JT, Bare O, Lerin C, Kim SH, Mostoslavsky R, Alt FW, Wu Z, Puigserver P. 2007. Metabolic control of muscle mitochondrial function and fatty acid oxidation through SIRT1/PGC-1 $\alpha$. EMBO J 26: 1913-1923.

Grant PA, Duggan L, Cote J, Roberts SM, Brownell JE, Candau R, Ohba R, Owen-Hughes T, Allis CD, Winston F, et al. 1997. Yeast Gcn5 functions in two multisubunit complexes to acetylate nucleosomal histones: Characterization of an Ada complex and the SAGA (Spt/Ada) complex. Genes Dev 11: 1640-1650.

Grant PA, Eberharter A, John S, Cook RG, Turner BM, Workman JL. 1999. Expanded lysine acetylation specificity of Gen5 in native complexes. J Biol Chem 274: 5895-5900.

Hallows WC, Lee S, Denu JM. 2006. Sirtuins deacetylate and activate mammalian acetyl-CoA synthetases. Proc Natl Acad Sci 103: 10230-10235. 
Hallows WC, Yu W, Smith BC, Devries MK, Ellinger JJ, Someya S, Shortreed MR, Prolla T, Markley JL, Smith LM, et al. 2011. Sirt3 promotes the urea cycle and fatty acid oxidation during dietary restriction. Mol Cell 41: 139-149.

Herranz D, Serrano M. 2010. SIRT1: Recent lessons from mouse models. Nat Rev Cancer 10: 819-823.

Hirschey MD, Shimazu T, Goetzman E, Jing E, Schwer B, Lombard DB, Grueter CA, Harris C, Biddinger S, Ilkayeva OR, et al. 2010. SIRT3 regulates mitochondrial fatty-acid oxidation by reversible enzyme deacetylation. Nature 464: $121-125$.

Huang JY, Hirschey MD, Shimazu T, Ho L, Verdin E. 2010. Mitochondrial sirtuins. Biochim Biophys Acta 1804: 16451651.

Imai S, Armstrong CM, Kaeberlein M, Guarente L. 2000a. Transcriptional silencing and longevity protein Sir2 is an NADdependent histone deacetylase. Nature 403: 795-800.

Imai S, Johnson FB, Marciniak RA, McVey M, Park PU, Guarente L. 2000b. Sir2: An NAD-dependent histone deacetylase that connects chromatin silencing, metabolism, and aging. Cold Spring Harb Symp Quant Biol 65: 297-302.

Jeninga EH, Schoonjans K, Auwerx J. 2010. Reversible acetylation of PGC-1: Connecting energy sensors and effectors to guarantee metabolic flexibility. Oncogene 29: 4617-4624.

Kaeberlein M, McVey M, Guarente L. 1999. The SIR2/3/4 complex and SIR2 alone promote longevity in Saccharomyces cerevisiae by two different mechanisms. Genes Dev 13: $2570-2580$

Kim SC, Sprung R, Chen Y, Xu Y, Ball H, Pei J, Cheng T, Kho Y, Xiao H, Xiao L, et al. 2006. Substrate and functional diversity of lysine acetylation revealed by a proteomics survey. Mol Cell 23: 607-618.

Laxman S, Tu BP. 2010. Systems approaches for the study of metabolic cycles in yeast. Curr Opin Genet Dev 20: 599-604.

Lee KK, Workman JL. 2007. Histone acetyltransferase complexes: One size doesn't fit all. Nat Rev Mol Cell Biol 8: 284-295.

Lerin C, Rodgers JT, Kalume DE, Kim SH, Pandey A, Puigserver P. 2006. GCN5 acetyltransferase complex controls glucose metabolism through transcriptional repression of PGC-1 $\alpha$. Cell Metab 3: 429-438.

Lin YY, Lu JY, Zhang J, Walter W, Dang W, Wan J, Tao SC, Qian J, Zhao Y, Boeke JD, et al. 2009. Protein acetylation microarray reveals that NuA4 controls key metabolic target regulating gluconeogenesis. Cell 136: 1073-1084.

Luo J, Nikolaev AY, Imai S, Chen D, Su F, Shiloh A, Guarente L, Gu W. 2001. Negative control of p53 by Sir $2 \alpha$ promotes cell survival under stress. Cell 107: 137-148.

Masumoto H, Hawke D, Kobayashi R, Verreault A. 2005. A role for cell-cycle-regulated histone $\mathrm{H} 3$ lysine 56 acetylation in the DNA damage response. Nature 436: 294-298.

Michan S, Sinclair D. 2007. Sirtuins in mammals: Insights into their biological function. Biochem J 404: 1-13.

Moore M, Jackson V, Sealy L, Chalkley R. 1979. Comparative studies on highly metabolically active histone acetylation. Biochim Biophys Acta 561: 248-260.

Picard F, Kurtev M, Chung N, Topark-Ngarm A, Senawong T, Machado De Oliveira R, Leid M, McBurney MW, Guarente L. 2004. Sirt1 promotes fat mobilization in white adipocytes by repressing PPAR-gamma. Nature 429: 771-776.

Polevoda B, Sherman F. 2002. The diversity of acetylated proteins. Genome Biol 3: reviews0006.

Polevoda B, Sherman F. 2003. N-terminal acetyltransferases and sequence requirements for $\mathrm{N}$-terminal acetylation of eukaryotic proteins. J Mol Biol 325: 595-622.

Puigserver P, Wu Z, Park CW, Graves R, Wright M, Spiegelman BM. 1998. A cold-inducible coactivator of nuclear receptors linked to adaptive thermogenesis. Cell 92: 829-839.

Rine J, Herskowitz I. 1987. Four genes responsible for a position effect on expression from HML and HMR in Saccharomyces cerevisiae. Genetics 116: 9-22.

Rodgers JT, Lerin C, Haas W, Gygi SP, Spiegelman BM, Puigserver P. 2005. Nutrient control of glucose homeostasis through a complex of PGC-1 $\alpha$ and SIRT1. Nature 434: $113-118$

Rogina B, Helfand SL. 2004. Sir2 mediates longevity in the fly through a pathway related to calorie restriction. Proc Natl Acad Sci 101: 15998-16003.

Saxholm HJ, Pestana A, O'Connor L, Sattler CA, Pitot HC. 1982. Protein acetylation. Mol Cell Biochem 46: 129-153.

Shi L, Sutter BM, Ye X, Tu BP. 2010. Trehalose is a key determinant of the quiescent metabolic state that fuels cell cycle progression upon return to growth. Mol Biol Cell 21: $1982-1990$.

Sinclair DA, Guarente L. 1997. Extrachromosomal rDNA circles-A cause of aging in yeast. Cell 91: 1033-1042.

Srere PA. 1959. The citrate cleavage enzyme. I. Distribution and purification. J Biol Chem 234: 2544-2547.

Takahashi H, McCaffery JM, Irizarry RA, Boeke JD. 2006. Nucleocytosolic acetyl-coenzyme a synthetase is required for histone acetylation and global transcription. Mol Cell 23: $207-217$.

Tanner KG, Trievel RC, Kuo MH, Howard RM, Berger SL, Allis CD, Marmorstein R, Denu JM. 1999. Catalytic mechanism and function of invariant glutamic acid 173 from the histone acetyltransferase GCN5 transcriptional coactivator. $J$ Biol Chem 274: 18157-18160.

Tanner KG, Landry J, Sternglanz R, Denu JM. 2000. Silent information regulator 2 family of NAD-dependent histone/ protein deacetylases generates a unique product, 1- $O$-acetylADP-ribose. Proc Natl Acad Sci 97: 14178-14182.

Tanno M, Sakamoto J, Miura T, Shimamoto K, Horio Y. 2007. Nucleocytoplasmic shuttling of the $\mathrm{NAD}^{+}$-dependent histone deacetylase SIRT1. J Biol Chem 282: 6823-6832.

Tanny JC, Moazed D. 2001. Coupling of histone deacetylation to NAD breakdown by the yeast silencing protein Sir2: Evidence for acetyl transfer from substrate to an NAD breakdown product. Proc Natl Acad Sci 98: 415-420.

Tissenbaum HA, Guarente L. 2001. Increased dosage of a sir-2 gene extends lifespan in Caenorhabditis elegans. Nature 410: 227-230.

Tu BP. 2010. Ultradian metabolic cycles in yeast. Methods Enzymol 470: 857-866.

Tu BP, McKnight SL. 2006. Metabolic cycles as an underlying basis of biological oscillations. Nat Rev Mol Cell Biol 7: 696-701.

Tu BP, Kudlicki A, Rowicka M, McKnight SL. 2005. Logic of the yeast metabolic cycle: Temporal compartmentalization of cellular processes. Science 310: 1152-1158.

Tu BP, Mohler RE, Liu JC, Dombek KM, Young ET, Synovec RE, McKnight SL. 2007. Cyclic changes in metabolic state during the life of a yeast cell. Proc Natl Acad Sci 104: $16886-16891$.

Vander Heiden MG, Cantley LC, Thompson CB. 2009. Understanding the Warburg effect: The metabolic requirements of cell proliferation. Science 324: 1029-1033.

Vaziri H, Dessain SK, Ng Eaton E, Imai SI, Frye RA, Pandita TK, Guarente L, Weinberg RA. 2001. hSIR2(SIRT1) functions as an NAD-dependent p53 deacetylase. Cell 107: $149-159$.

Wang C, Chen L, Hou X, Li Z, Kabra N, Ma Y, Nemoto S, Finkel T, Gu W, Cress WD, et al. 2006. Interactions between E2F1 and SirT1 regulate apoptotic response to DNA damage. Nat Cell Biol 8: 1025-1031.

Wang Q, Zhang Y, Yang C, Xiong H, Lin Y, Yao J, Li H, Xie L, Zhao W, Yao Y, et al. 2010. Acetylation of metabolic enzymes coordinates carbon source utilization and metabolic flux. Science 327: 1004-1007.

Wellen KE, Hatzivassiliou G, Sachdeva UM, Bui TV, Cross JR, Thompson CB. 2009. ATP-citrate lyase links cellular metabolism to histone acetylation. Science 324: 1076-1080.

Wu Z, Puigserver P, Andersson U, Zhang C, Adelmant G, Mootha V, Troy A, Cinti S, Lowell B, Scarpulla RC, et al. 1999. Mechanisms controlling mitochondrial biogenesis and respiration through the thermogenic coactivator PGC-1. Cell 98: $115-124$ 
Xu F, Zhang K, Grunstein M. 2005. Acetylation in histone H3 globular domain regulates gene expression in yeast. Cell 121: $375-385$.

Yoon JC, Puigserver P, Chen G, Donovan J, Wu Z, Rhee J, Adelmant G, Stafford J, Kahn CR, Granner DK, et al. 2001. Control of hepatic gluconeogenesis through the transcriptional coactivator PGC-1. Nature 413: 131-138.

Zhang J, Sprung R, Pei J, Tan X, Kim S, Zhu H, Liu CF, Grishin NV, Zhao Y. 2009. Lysine acetylation is a highly abundant and evolutionarily conserved modification in Escherichia coli. Mol Cell Proteomics 8: 215-225.

Zhang Z, Tan M, Xie Z, Dai L, Chen Y, Zhao Y. 2011. Identification of lysine succinylation as a new post-translational modification. Nat Chem Biol 7: 58-63.

Zhao S, Xu W, Jiang W, Yu W, Lin Y, Zhang T, Yao J, Zhou L, Zeng Y, Li H, et al. 2010. Regulation of cellular metabolism by protein lysine acetylation. Science 327: 10001004 . 


\section{$\$_{\text {CSH\& }}^{\infty}$ Cold Spring Harbor Symposia SYMPOSIA On Quantitative Biology}

\section{On Acetyl-CoA as a Gauge of Cellular Metabolic State}

L. Cai and B.P. Tu

Cold Spring Harb Symp Quant Biol 2011 76: 195-202 originally published online September 6, 2011

Access the most recent version at doi:10.1101/sqb.2011.76.010769

References This article cites 71 articles, 27 of which can be accessed free at: http://symposium.cshlp.org/content/76/195.full.html\#ref-list-1

\section{License}

Email Alerting

Receive free email alerts when new articles cite this article - sign up in

Service the box at the top right corner of the article or click here. 